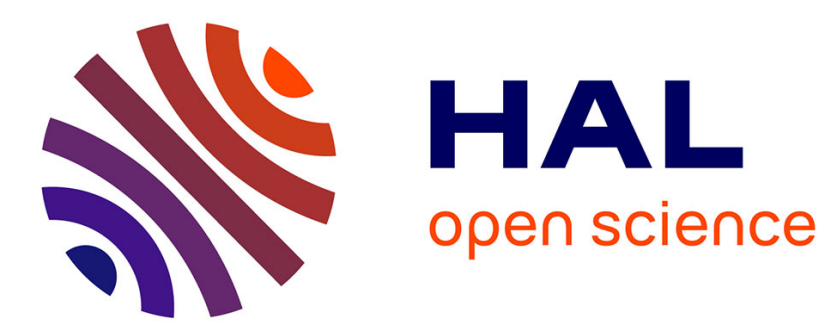

\title{
Exploration de formes géométriques par le toucher
}

Thomas Pietrzak, Andrew Crossan, Stephen A. Brewster, Benoît Martin, Isabelle Pecci

\section{To cite this version:}

Thomas Pietrzak, Andrew Crossan, Stephen A. Brewster, Benoît Martin, Isabelle Pecci. Exploration de formes géométriques par le toucher. Proceedings of the AFIHM Conférence Francophone sur l'interaction Homme-Machine (IHM 2009), 2009, Grenoble, France. 10.1145/1629826.1629866 . hal00671482

\section{HAL Id: hal-00671482 \\ https://hal.inria.fr/hal-00671482}

Submitted on 17 Feb 2012

HAL is a multi-disciplinary open access archive for the deposit and dissemination of scientific research documents, whether they are published or not. The documents may come from teaching and research institutions in France or abroad, or from public or private research centers.
L'archive ouverte pluridisciplinaire HAL, est destinée au dépôt et à la diffusion de documents scientifiques de niveau recherche, publiés ou non, émanant des établissements d'enseignement et de recherche français ou étrangers, des laboratoires publics ou privés. 


\title{
Exploration de formes géométriques par le toucher
}

\author{
Thomas Pietrzak $\dagger$ Andrew Crossan $\ddagger$ Stephen A. Brewster $\ddagger \quad$ Benoît Martin $\dagger \quad$ Isabelle Pecci $\dagger$ \\ $\dagger$ LITA, UFR MIM \\ île du Saulcy \\ 57000, Metz, France \\ \{pietrzak, benoit.martin, pecci\}, @ univ-metz.fr \\ $\ddagger 17$ Lilybank Gardens \\ Université de Glasgow \\ Glasgow, G12 8RZ, UK \\ \{ac, stephen\}@dcs.gla.ac.uk
}

\begin{abstract}
RESUME
Nous proposons une nouvelle technique pour aider des personnes à explorer des formes géométriques sans l'aide de la vision. Cette technique est basée sur un guidage utilisant des icônes directionnelles affichées sur une matrice de picots. Il s'agit d'une alternative à la méthode usuelle consistant à lever les picots correspondant aux pixels sombres autour du curseur. Dans cet article nous comparons deux méthodes d'exploration de formes géométriques avec notre nouvelle technique : en conditions unimanuelle et bimanuelle. D'après nos résultats, les utilisateurs ont fait peu d'erreurs dans les deux conditions. De plus les résultats montrent une équivalence des deux techniques en terme de temps de réponse et de confiance des utilisateurs en leur réponse.
\end{abstract}

MOTS CLES : Interaction tactile, Tactons, Géométrie, Interaction non-visuelle.

\begin{abstract}
We propose a new technique to help people to explore geometric shapes without vision. This technique is based on a guidance using directional cues with a pin array. This is an alternative to the usual technique that consists of raising the pins corresponding to dark pixels around the cursor. In this paper we compare the exploration of geometric shapes with our new technique in unimanual and bimanual conditions. According to our results, the users made few errors in both conditions. Moreover the results show an equivalence for both techniques in answer time and users' confidence in their answer.
\end{abstract}

CATEGORIES AND SUBJECT DESCRIPTORS: H.5.2. Haptic I/O, H.1.2 Human Factors, I.3.6 Interaction Techniques

Permission to make digital or hard copies of all or part of this work for personal or classroom use is granted without fee provided that copies are not made or distributed for profit or commercial advantage and that copies bear this notice and the full citation on the first page. To copy otherwise, to republish, to post on servers or to redistribute to lists, requires prior specific permission and/or a fee.

IHM 2009, 13-16 Octobre 2009, Grenoble, France

Copyright 2009 ACM 978-1-60558-461-4/09/10 ...\$5.00.
GENERAL TERMS: Design, Human Factors, Performance.

KEYWORDS: Tactile interaction, Tactons, non-visual interaction, geometric shapes.

\section{INTRODUCTION}

De nombreux domaines enseignés à l'école ont souvent recours à des données structurées telles que des schémas. Ceux-ci sont des représentations essentiellement visuelles, et sont difficiles à interpréter pour un enfant possédant une déficience visuelle. Les enfants possédant une vision résiduelle peuvent éventuellement utiliser des schémas agrandis, mais ceux qui sont aveugles ou qui ont une faible vision résiduelle doivent utiliser un autre sens. Le papier en relief est très répandu à cet usage. Les enfants peuvent explorer les schémas sur papier en relief en déplaçant leurs doigts sur le papier et sentir les parties embossées de l'image [5]. Cependant cette technique procure une représentation statique des schémas, et ne profite pas des avantages offerts par les systèmes informatisés. Les grands afficheurs braille peuvent potentiellement fournir une fonctionnalité similaire sur un système informatisé, mais leur coût est généralement prohibitif [3]. De plus, leur taille n'atteint pas celle d'une feuille A4. Le but de nos recherches est d'identifier des techniques qui permettent d'apporter des représentations accessibles pour des données structurées telles que les formes ou les schémas, en gardant les avantages de l'approche technologique et d'un coût modéré.

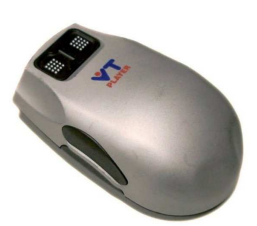

Figure 1 : Souris VTPlayer

La souris VTPlayer (figure 1) est un exemple d'afficheur de ce type. Elle possède deux matrices de $4 \times 4$ picots sur le dessus. Elle a déjà été utilisée pour afficher des icônes tactiles, et en ce sens a montré qu'elle était un bon moyen de transmettre des informations [8, 7]. Nous 
nous intéressons à deux aspects concernant l'affichage de schémas à l'aide du toucher. La première est la façon dont les informations sont présentées, et la seconde est la façon dont les schémas sont explorés. Dans cet article nous proposons une nouvelle méthode de présentation, que nous expérimenterons à l'aide de deux méthodes d'exploration.

\section{MÉTHODES DE PRÉSENTATION ET D'EXPLORATION}

La méthode courante pour afficher des schémas à l'aide d'une matrice de picots est une méthode de traduction visuelle, qui est une simple correspondance des pixels sombres en picots levés. Il a été montré que cette méthode est inefficace [4], à moins qu'elle ne soit agrémentée d'un système de guidage [9, 10].

Notre nouvelle méthode consiste à guider les utilisateurs sur la forme afin de les aider à la parcourir complètement. Elle est basée sur une représentation vectorielle de la forme à explorer. La forme est décomposée en segments sur lesquels l'utilisateur est guidé à l'aide de Tactons. Les Tactons sont des signaux tactiles structurés qui permettent de transmettre des informations [1]. Nous utilisons des Tactons construits à partir d'une matrice de $4 \times 4$ picots d'une souris VTPlayer, qui ont déjà permis de représenter 4 et 8 directions (figure 2) dans des études précédentes [2, 8]. Les informations sont représentées à l'aide des différents paramètres de construction des Tactons. Les Tactons statiques sont définis par un simple motif, alors que les tactons dynamiques sont des animations définis par la séquence de motifs affichés ainsi que par la vitesse de lecture.

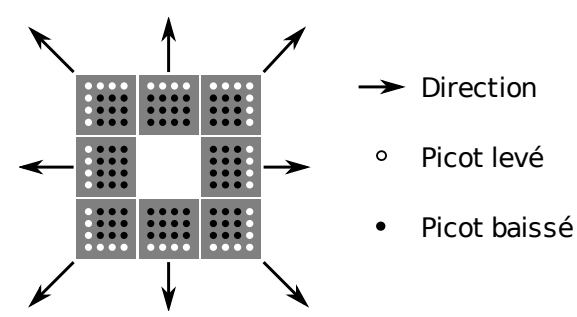

Figure 2 : Motifs utilisés pour représenter 8 directions

Une étude préliminaire sur l'exploration de formes à l'aide de Tactons à matrices de picots en exploration unimanuelle [6] montre que les utilisateurs parviennent à explorer des formes à l'aide de cette technique. Cependant les seules informations données par les Tactons étaient les directions. L'étape suivante consiste à améliorer ce système en ajoutant plus d'informations. D'après nos études fondamentales sur les Tactons, il est possible de transmettre plusieurs informations par Tacton, en faisant varier plusieurs de ses paramètres [7]. Par exemple, les Tactons dynamiques de type clignotant alternent un motif avec des picots levés et un autre motif sans picot levé, ces deux motifs étant affichées pendant la même durée.

Dans notre cas nous pouvons afficher une information à l'aide du motif, et une autre à l'aide de la vitesse de cli- gnotement. Le prochain sommet à atteindre est appelé la cible. Nous utilisons toujours le motif pour représenter sa direction, et nous utilisons la vitesse de clignotement pour représenter sa distance. Nos études préliminaires ont montré que des utilisateurs parvenaient à distinguer 3 vitesses de clignotement avec une grande exactitude [7]. Nous avons choisi d'associer la vitesse de clignotement élevée à une faible distance et inversement, par analogie avec signal de sonar. Enfin nous utilisons aussi une simple association binaire sur la deuxième matrice, qui se trouve sous le majeur de la main, afin de signaler à l'utilisateur si il se trouve sur la forme ou non.

Les segments ont une épaisseur, fixée après une étude pilote. La cible est représentée par un demi-cercle dont le rayon est égal à l'épaisseur du segment (figure 3). L'utilisateur est guidé d'un segment à l'autre en boucle, c'està-dire que le trajet est imposé mais qu'il peut explorer la forme autant de fois qu'il le souhaite. Le Tacton est affiché sous l'index de la main, et ses caractéristiques dépendent de la position de l'utilisateur par rapport au segment. Si l'utilisateur est sur le segment, le Tacton indique la cible. Sinon il indique le point le plus proche sur le segment (projection orthogonale ou extrémité).

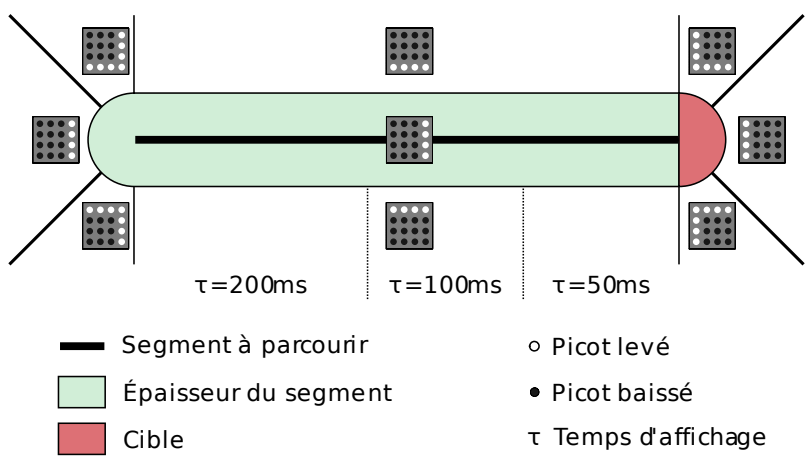

Figure 3 : Guidage sur un segment

Nous allons étudier deux modes d'exploration : unimanuel et bimanuel. En mode unimanuel, le participant utilise sa main dominante à la fois pour explorer la forme, et pour ressentir les informations tactiles (comme dans [4]). L'exploration se fait donc uniquement à l'aide de la souris VTPlayer. En mode bimanuel, l'utilisateur explore la forme avec une tablette graphique dans sa main dominante, et ressent les Tactons sur la VTPlayer qu'il tient dans la seconde main et qui ne bouge pas (comme dans [9, 10]). L'intérêt de la première méthode est qu'elle fournit des informations kinesthésiques venant du mouvement d'exploration sur la même partie du corps que celle qui donne les sensations tactiles. L'intérêt de la seconde méthode est qu'elle offre une solution alternative pour les utilisateurs aveugles, pour qui des études précédentes ont révélé que l'utilisation d'une souris peut être problématique [4]. En effet, l'utilisation de coordonnées absolues pourrait permettre à garder un point de repère lors d'une exploration non-visuelle. 


\section{EXPÉRIENCE}

\section{Méthodologie}

Dans cette étude préliminaire, nous allons comparer deux méthodes d'exploration pour notre nouvelle méthode de présentation. La tâche consistait à identifier une série de formes le plus exactement possible et le plus rapidement possible.

Huit utilisateurs voyants occultés ont participé à cette expérience. Ils étaient âgés de 26 à 28 ans, aucun d'entre eux ne souffrant de problèmes connus de perception tactile. Tous sont des utilisateurs réguliers d'ordinateurs, mais aucun n'a d'expérience approfondie dans l'usage de la tablette graphique. Les utilisateurs ne portaient pas de casque avec un bruit blanc car nous considérons que les informations sonores données par le déplacement des picots font partie de l'usage courant de l'application. Pour une étude sur l'efficacité de la technique d'interaction haptique, le lecteur pourra se référer à [7].

La première moitié des utilisateurs explore les formes avec la souris VTPlayer, et la seconde moitié utilise la tablette graphique. Chaque participant commençait par explorer une forme en guise d'entrainement jusqu'à ce qu'il soit à l'aise avec le système. Cette phase durait typiquement 5 minutes. Ensuite il devait explorer les 10 formes à reconnaître, avec un temps maximum d'exploration de 3 minutes fixé après une étude pilote. Pour chaque forme l'utilisateur devait désigner la forme qu'il avait exploré parmi une représentation visuelle de toutes les formes possibles. Parmi les formes il y avait des carrés, des triangles et d'autres polygones simples (figure 4). Il devait aussi donner un indice de confiance en sa réponse entre 1 et 7 . De plus le système enregistrait la trajectoire, ainsi que le temps d'exploration.
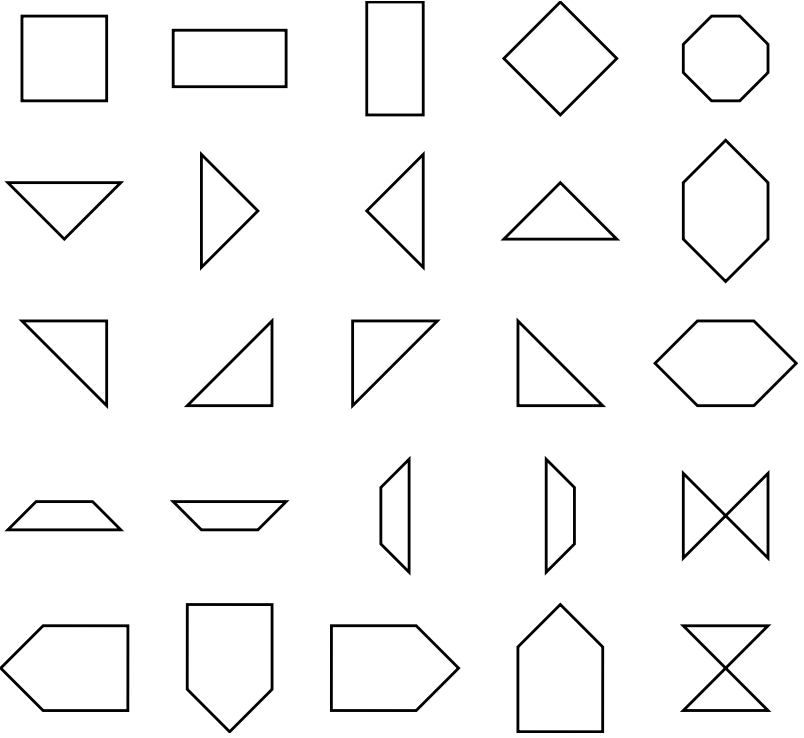

Figure 4 : Formes possibles

Nous émettons l'hypothèse que les utilisateurs explorant en mode unimanuel feront moins d'erreurs, répondront plus rapidement et seront plus confiants en leur réponse que ceux explorant en mode bimanuel. En effet, nous pensons que l'utilisation d'une main différente pour l'exploration et pour acquérir les information est une source de confusion pour les utilisateurs.

\section{Résultats}

Dans un premier temps nous remarquons un taux d'erreur encourageant : les utilisateurs explorant en mode unimanuel on donné au total 4 réponses erronées sur 40 , et ceux ayant exploré en mode bimanuel ont donné 8 mauvaises réponses sur 40.

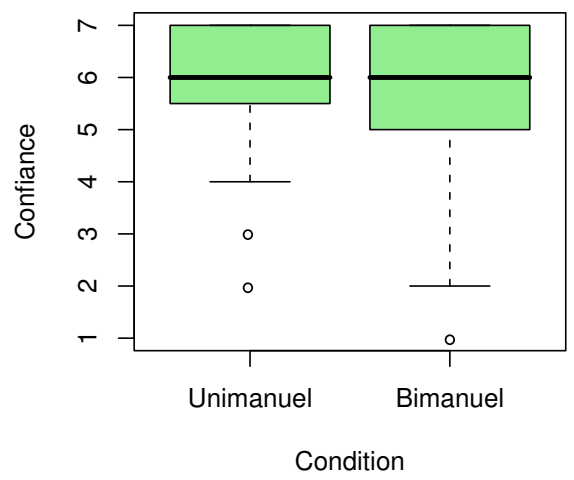

Figure 5 : Confiance en la réponse

La confiance des utilisateurs en leur réponse est représentée sur la figure 5 Les utilisateurs ayant exploré en mode unimanuel ont donné un indice de confiance moyen de 5,85/7 $(\sigma=1,28)$ et ceux ayant exploré en mode bimanuel ont donné un indice de confiance moyen de 5,6/7 $(\sigma=1,62)$. Le test de la somme des rangs de Wilcoxon ne permet pas de trouver de différence significative entre les deux conditions $(W=878, p=0,43)$.

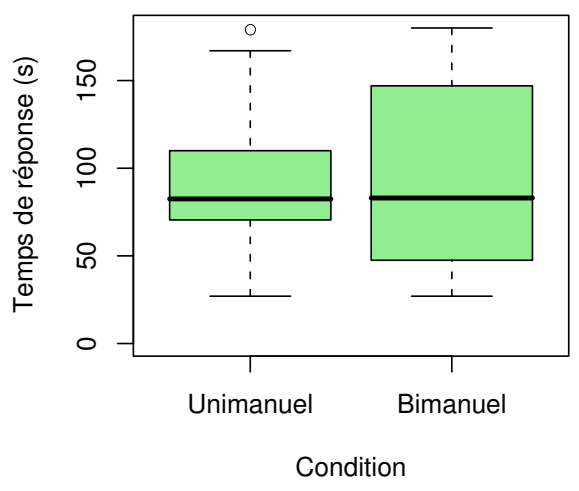

Figure 6: Temps de réponse

Les temps de réponse des utilisateurs sont représentés sur la figure 6. Nous constatons que les utilisateurs de la condition unimanuelle ont répondu avec une moyenne de 95,95s ( $\sigma=42,77 \mathrm{~s})$ et les utilisateurs de la condition bimanuelle ont répondu avec une moyenne de $93,45 \mathrm{~s}$ $(\sigma=52,54 s)$. Le test de la somme des rangs de Wilcoxon ne permet pas de différencier ces deux conditions $(W=881.5, p=0,43)$. 


\section{Discussion}

Les taux d'erreur sont relativement faibles, d'autant plus que cette méthode reste perfectible. En outre le nombre d'informations à assimiler est important pour un novice. Plusieurs utilisateurs ont avoué être perdus au début de la session et se sont sentis plus à l'aise à la fin. À l'avenir nous nous efforcerons d'introduire les différentes informations de manière plus progressive.

Nos statistiques ne nous ont pas permis de trouver de différences significatives entre les résultats des utilisateurs sur les deux conditions, que ce soit en terme de temps de réponse ou de confiance en la réponse. Nous devons donc rejeter notre hypothèse selon laquelle les utilisateurs auraient de meilleures performances en mode unimanuel. Cependant nous notons que deux utilisateurs explorant en mode bimanuel ont signalé avoir été perturbés par le fait d'explorer avec une main et de recevoir les informations sur l'autre main.

Les résultats de notre nouvelle méthode sont encourageants de manière générale car les utilisateurs ont fait peu d'erreurs avec peu d'entrainement. Il serait intéressant d'étudier l'impact d'un entrainement plus poussé sur les résultats des utilisateurs. De plus nous sommes conscients que les résultats pourraient différer avec des utilisateurs déficients visuels. Cependant de tels utilisateurs sont rares, donc nous préférons leur faire tester l'application finale.

\section{CONCLUSION}

Nous avons proposé dans cet article une nouvelle méthode de présentation de formes géométriques. Dans cette étude préliminaire nous avons testé la différence entre deux modes d'exploration avec cette méthode : en unimanuel et en bimanuel. Les résultats ont montré que les utilisateurs parviennent à explorer efficacement des formes géométriques de manière non-visuelle à l'aide de notre méthode, dans le sens où le nombre d'erreurs et le temps d'exploration étaient raisonnablement faibles.

Nous n'avons décelé aucune différence significative entre les performances en unimanuel et en bimanuel. Ceci nous encourage à nous concentrer sur le mode bimanuel qui utilise une tablette graphique pour l'exploration et la souris VTPlayer en tant qu'afficheur Braille. La raison est que la tablette graphique utilise des coordonnées absolues, ce qui est plus pratique pour les utilisateurs aveugles que les coordonnées relatives d'une souris. Ainsi nous espérons contourner les problèmes soulevés par d'autres études [4].

Dans nos prochains travaux, nous allons comparer les performances de cette nouvelle méthode avec celles de la méthode usuelle qui consiste à lever les picots correspondant aux pixels sombres autour du curseur. Nous étudierons aussi les performances d'utilisateurs déficients visuels sur ce système.

\section{REMERCIEMENTS}

Nous tenons à remercier les utilisateurs qui nous ont gracieusement accordé un peu de leur temps pour ces tests.

\section{BIBLIOGRAPHIE}

1. Brewster, S. A., and Brown, L. M. Non-visual information display using tactons. In $\mathrm{CHI}$ '04 : Extended abstracts on Human factors in computing systems, pages 787-788, Vienna, Austria, Apr. 2004. ACM Press.

2. Crossan, A., and Brewster, S. A. Two-handed navigation in a haptic virtual environment. In $\mathrm{CHI}$ '06 : Proceedings of the SIGCHI conference on Human factors in computing systems, Montréal, Québec, Canada, Apr. 2006. ACM Press.

3. Fujiyoshi, M., Fujiyoshi, A., Ohtake, N., Yamaguchi, K., and Teshima, Y. The development of a universal design tactile graphics production system bplot2. In ICCHP 2008 : proceedings of the 11th conference ICCHP, pages 938-945, Linz, Austria, July 2008.

4. Jansson, G., and Pedersen, P. Obtaining geographical information from a virtual map with a haptic mouse. In International Cartographic Conference, La Coruña, Spain, July 2005.

5. Lévesque, V. Blindness, technology and haptics. Technical report, McGill University, Montréal, Québec, Canada, 2005.

6. Noble, N., and Martin, B. Shape discovering using tactile guidance. In Proceeding of the 6th International Conference EuroHaptics 2006, Paris, France, July 2006.

7. Pietrzak, T., Crossan, A., Brewster, S. A., Martin, B., and Pecci, I. Creating usable pin array tactons for non-visual information. IEEE Transactions on Haptics, 2(2), 2009. 12 pages.

8. Pietrzak, T., Pecci, I., and Martin, B. Static and dynamic tactile directional cues experiments with VTPlayer mouse. In Proceeding of the 6th International Conference EuroHaptics 2006, pages 63-68, Paris, France, July 2006.

9. Wall, S. A., and Brewster, S. A. Tac-tiles : multimodal pie charts for visually impaired users. In 4th Nordic Conference on Human-Computer Interaction, pages 9-18, Oslo, Norway, Oct. 2006. ACM Press.

10. Ziat, M., Gapenne, O., Stewart, J., and Lenay, C. Haptic recognition of shapes at different scales : A comparison of two methods of interaction. Interacting with Computers, 19(1) :121-132, Jan. 2007. 